\title{
Recursive estimation of the parts production process quality indicator
}

\author{
Oleg Filipovich ${ }^{1, *}$, Vadim Kopp ${ }^{1}$, Nataliya Voloshina ${ }^{1}$, and Alexander Bokhonsky ${ }^{1}$ \\ ${ }^{1}$ Sevastopol State University, Sevastopol, Russian Federation
}

\begin{abstract}
Consideration is given to a mathematical representation for manufacturing of batch parts on a metal-cutting machine tool. Linear dimensions of machined parts are assumed to be the major quality indicator, deviation from these dimensions is determined by size setting of machine tool and ensemble of random factors. It is allowed to have absolutely precise pre-setting of machine tool, effects from setup level offsetting due to deformation in process equipment on the specified indicator are disregarded. Consideration is given to factors which affect the tool wear, with two definitions of tool wear being provided. Reasons for development of random error in processing, dependence of measurement results on error as well as distribution laws and some parameters of random values are provided. To evaluate deviation of size setting value in each cycle, it is proposed to apply a recursive algorithm in description of investigated dynamic discrete process in the space state. Kalman filter equations are used in description of process model by means of first-order difference equations. The algorithm of recursive estimation is implemented in the mathematical software Maple. Simulation results which prove effectiveness of algorithm application to investigate the given dynamic system are provided. Variants of algorithm application and opportunities of further research are proposed.
\end{abstract}

Consideration is given to machining of part batch $N$ made with a cutting machine tool. A cycle is understood as completion of total operational sequence for each part made on a single piece of production machinery.

Mathematical description of part fabrication process seen from the aspect of achieving a required quality indicator is presented in detail in [1]. The linear size at some fixed section is selected as a major criterion of machining accuracy while errors of part shape are neglected. Achievement of required indicator is associated with a complex fabrication process under conditions of dynamically non-deterministic environment. One of possible objectives here is to obtain a forecast for deviation value of dimensional parameter with the maximum feasible precision. The purpose of this study is to apply known algorithms to procure recursive estimates for the mentioned indicator.

In real-life conditions any manufacturing process is realized as affected by multiple random disturbances which depend on the strength and oscillations of workpiece allowance, deformations during machining, value of cutting tool wear etc. Taking this fact into account, the quality indicator value (deviation of linear size from some nominal value) can be written as:

$$
x_{k}=\overline{x_{k}}+\xi_{k},
$$

where $\overline{x_{k}}-$ value of machine tool size setting in $k$-n cycle, which represents the total of determined trend and random functional component of processing error; $\xi_{k}-$ random departure of part dimensions from the setting level.

Drift of setting level in $k$-n cycle of manufacturing process can be expressed in the following form:

$$
\overline{x_{k}}=x_{0}+\mu_{k}+l_{k},
$$

where

$x_{0}$ - initial offset in the setting level;

$\mu_{k}$ - offset in the setting level due to deformation of manufacturing machinery;

$l_{k}$ - offset in the setting level due to wear of cutting tool.

To simplify further explication let us assume that the setup was absolutely accurate to match zero level, i.e. $x_{0}=0$.

There are thermal and mechanical deformations of manufacturing system to be distinguished. Thermal deformations depend on the design of separate machine tool assemblies, amount of coolant and degree of its heating, extension of dimension chains, cutting tool characteristics and bluntness degree, feed, cutting speed and depth, material and workpiece size, ambient temperature, friction forces etc. Mechanical deformations depend on stiffness of joints, cutting depth, feed rate, on size, hardness and quality of a workpiece, quality and size of a cutting tool, cutting speed, degree of

\footnotetext{
* Corresponding author: ophisl@yandex.ru
} 
heating in cutting tool assemblies, lubricant etc. When considering the case of piece machining on metal-cutting machine tools with disturbances related mostly to severe wear of cutting tool, we can neglect drifting of setting level due to deformation of manufacturing machinery, i.e. $\mu_{k}=0$.

Value of tool wear in each cycle of process depends on such factors as machining allowance value or material hardness, which are of random nature. Therefore, on the whole, wear is a random parameter whose value depends neither on values in preceding cycles nor on total wear. Let us consider two cases.

Case 1. If dispersion of this value is not significant, then the trend can be regarded as uniform and represented as linear function

$$
l_{k}=\alpha k,(k=\overline{1, N}),
$$

where

$\alpha$ - cutting tool wear rate which falls within one cycle.

In this case the value $l_{k}$ is a systematic component of error and a determined value.

Case 2. If dispersion of wear value in each cycle is significant, and the wear factor is prevalent in (1), then it can be regarded as a stochastic process with independent increments. The value $l_{k}$ in this case can be represented in the form of [2]

$$
l_{k}=\alpha k+\sum_{i=1}^{k} \gamma_{i}, \quad(k=\overline{1, N}),
$$

where

$\gamma_{i}$ - independent increment in $i$-n cycle (stochastic variable distributed in accordance with the Gaussian law with zero mathematical expectation and dispersion $\sigma_{\gamma}^{2}$ ).

There are random errors developed in each manufacturing process cycle during machining of parts which are known as "instantaneous" processing errors or purely random errors $\xi_{k}$. Their sources lie in nonhomogeneity of work pieces in their machining allowances and hardness, in non-homogeneity of cutting tool structure and in random changes of cutting modes. Salient feature of sequence $\left\{\xi_{k}\right\}$ formed actually by random errors of manufacturing process is mutual independence of values from each other. Research demonstrates that the random value $\xi_{k}$ is distributed independent of $k$ - with zero mathematical expectation, as a rule, while its dispersion retains constancy in sufficiently stable and debugged manufacturing process for a long period of time $[2,3,4]$.

By substituting (2) in (1), we obtain - with due account for made assumptions

$$
x_{k}=l_{k}+\xi_{k} .
$$

To determine the parameter $x_{k}$, it is measured after completion of each cycle by passive control system.
Results of $z_{k}$ measurements under certain assumptions can be expressed by equation

$$
z_{k}=x_{k}+\delta_{k},
$$

where

$\delta_{k}-$ measurement error.

On condition that systematic errors are missing or compensated let us determine the distribution law for the random component of measurement error. It was demonstrated in work [5] that the maximum differential entropy of continuous random variable constrained by finite limits provides for the composition law, which, depending on the dispersion value, manifests itself as truncated normal, uniform or bimodal. Let us assume that at certain dispersion restrictions the measurement error $\delta_{k}$ has the truncated normal distribution whose density function at zero mathematical expectation equals

$$
f(\delta)=\frac{1}{C \sigma_{\delta} \sqrt{2 \pi}} e^{-\frac{\delta^{2}}{2 \sigma_{\delta}^{2}}}, \delta \in[-a, a],
$$

where

$[-a, a]$ - limits of random value variation;

$\sigma_{\delta}, C$ - law parameters,

$$
C=\int_{-a}^{a} f(\delta) d \delta
$$

To solve the task of target indicator assessment, let us apply the recursive algorithm by describing the dynamic process under study in the state space form. Recursive algorithms of estimation (Kalman filter) became widespread when solving a number of identification, evaluation, filtration and control tasks [6...22]. Let us assume that the process model is described by first-order difference equations. In our case there is a given discrete scalar system

$$
\begin{aligned}
& x_{k+1}=x_{k}+l_{k}+\xi_{k} \\
& z_{k+1}=x_{k+1}+\delta_{k+1}
\end{aligned}
$$

where

$x_{k+1}$ and $x_{k}$ - values of quality indicator at $(k+1)$-n and $k-n$ cycles of manufacturing process respectively;

$l_{k}-$ a known value that determines evolution of system;

$\xi_{k}$ and $\delta_{k}$ - stationary random values whose distribution laws and moment characteristics were described earlier .

When determining the value $l_{k}$, let us assume that the value of tool wear has linear dependence on $k$ (case 1).

Let us assume that all random values are independent and the system is stable, fully controllable and observable. The Kalman filter equation for such system will be of the following form 


$$
\begin{aligned}
& \hat{x}_{k+1}=\hat{x}_{k}+K_{k+1}\left(z_{k+1}-\hat{x}_{k}\right), \\
& K_{k+1}=\frac{V_{\varepsilon k}+\sigma_{\xi}^{2}}{V_{\varepsilon k}+\sigma_{\xi}^{2}+\sigma_{\delta}^{2}}
\end{aligned}
$$

on initial conditions $\hat{x}_{0}=0, \sigma_{\xi 0}^{2}=0$,

where

$\hat{x}_{k+1}, \hat{x}_{k}$ - estimates of values $x_{k+1}$ and $x_{k}$;

$K_{k+1}$ - Kalman gain whose optimal value minimizes the RMS estimation error

$$
V_{\varepsilon k+1}=M\left[\varepsilon_{k+1}^{2}\right]=\frac{\sigma_{\delta}^{2}\left(V_{\varepsilon k}+\sigma_{\xi}^{2}\right)}{V_{\varepsilon k}+\sigma_{\xi}^{2}+\sigma_{\delta}^{2}},
$$

where

$M$ - symbol of mathematical expectation;

$\varepsilon_{k+1}$ - estimation error,

$$
\varepsilon_{k+1}=x_{k+1}-\hat{x}_{k+1} .
$$

The algorithm $(10 \ldots 12)$ is implemented in the Maple system with the following given data:
1. $N=20$ parts,
2. $\alpha=3 \cdot 10^{-6} \mathrm{~m} /$ cycle,
3. $\sigma_{\delta}=2 \cdot 10^{-6} \mathrm{~m}$,
4. $a=2 \sigma_{\delta}=4 \cdot 10^{-6} \mathrm{~m}$,
5. $\sigma_{\xi}=1 \cdot 10^{-6} \mathrm{~m}$

Results from incremental determining of values $x_{k}$, $z_{k}$ и $\hat{x}_{k}$ are summarized in Table 1.

Table 1. Simulation results

\begin{tabular}{|c|c|c|c|}
\hline $\begin{array}{c}\text { No. of } \\
\text { cycle, } k\end{array}$ & $\begin{array}{c}x_{k}, \\
10^{-6} \mathrm{~m}\end{array}$ & $\begin{array}{c}z_{k}, \\
10^{-6} \mathrm{~m}\end{array}$ & $\begin{array}{c}\hat{x}_{k}, \\
10^{-6} \mathrm{~m}\end{array}$ \\
\hline 1. & 0,0 & 1,2 & - \\
\hline 2. & 2,6 & 3,4 & 3,8 \\
\hline 3. & 5,0 & 5,4 & 6,2 \\
\hline 4. & 7,9 & 7,1 & 8,3 \\
\hline 5. & 11,4 & 9,9 & 10,7 \\
\hline 6. & 14,3 & 13,6 & 13,7 \\
\hline 7. & 17,2 & 14,4 & 15,8 \\
\hline 8. & 20,9 & 19,4 & 19,0 \\
\hline 9. & 24,0 & 24,9 & 23,2 \\
\hline 10. & 26,4 & 26,2 & 26,2 \\
\hline 11. & 29,5 & 27,8 & 28,6 \\
\hline 12. & 33,6 & 31,6 & 31,6 \\
\hline 13. & 35,9 & 34,1 & 34,4 \\
\hline 14. & 38,0 & 39,9 & 38,4 \\
\hline 15. & 40,6 & 39,1 & 40,5 \\
\hline 16. & 43,0 & 45,2 & 44,2 \\
\hline
\end{tabular}

\begin{tabular}{|l|l|l|l|}
\hline 17. & 45,1 & 46,6 & 46,9 \\
\hline 18. & 47,2 & 47,6 & 49,0 \\
\hline 19. & 50,2 & 49,9 & 51,2 \\
\hline 20. & 53,1 & 53,4 & 53,9 \\
\hline
\end{tabular}

Of special importance is comparison of value $\varepsilon_{k}$ and difference

$$
\Delta_{k}=x_{k}-z_{k}
$$

at every cycle. The plot that illustrates their change is displayed in Fig. 1.

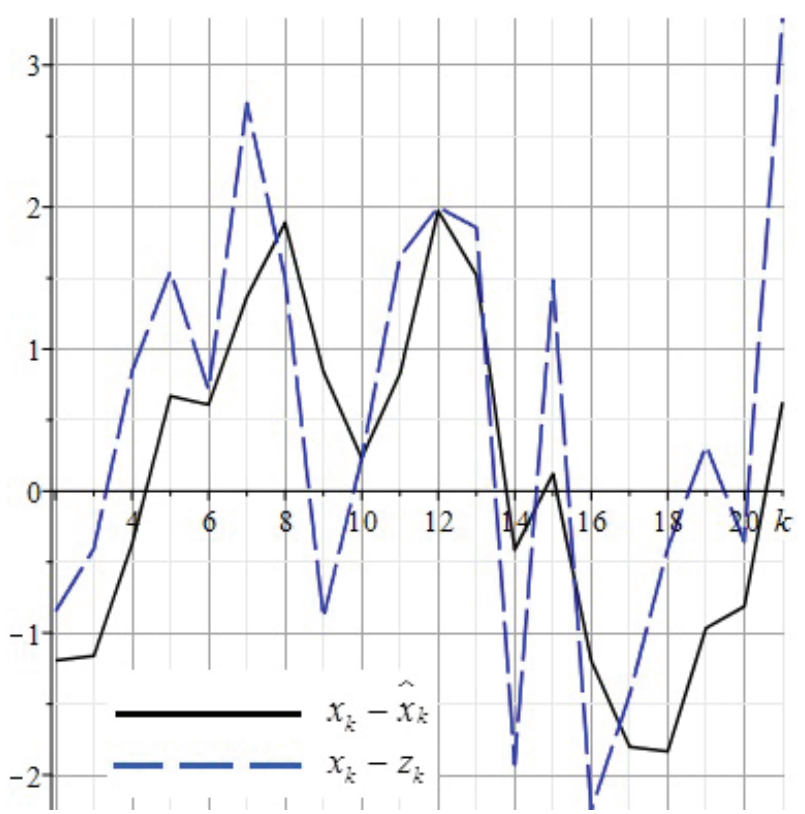

Fig. 1. Results from determining $\varepsilon_{k}$ and $\Delta_{k}$ values depending on the number of cycle $k$

As it can be seen from the results presented in Table 1 and in Fig.1, the mean relative value $\varepsilon_{k}$ does not exceed $2 \%$, which is a sufficiently good estimation in the framework of task to be solved. At the same time, the mean relative difference between $x_{k}$ and $z_{k}$ is approximately 1.5 times higher than this value, thus confirming the effectiveness of estimation. The estimation algorithm for this task is not divergent, the gain becomes stable fairly quickly (Fig.2).

The values of the function $V_{\varepsilon}(k)$ are displayed in Fig. 3. It can be seen that after 10 cycles this function goes to a certain limit.

The results obtained can be used to improve operation efficiency of data management and control systems installed on metal working machines, which provide for their independent setup and adjustment during operation. It is also proposed to apply this algorithm further on to evaluate probability indicators for batches composed of two various products and assembled jointly with the use of group interchangeability technique (selective assembly). 


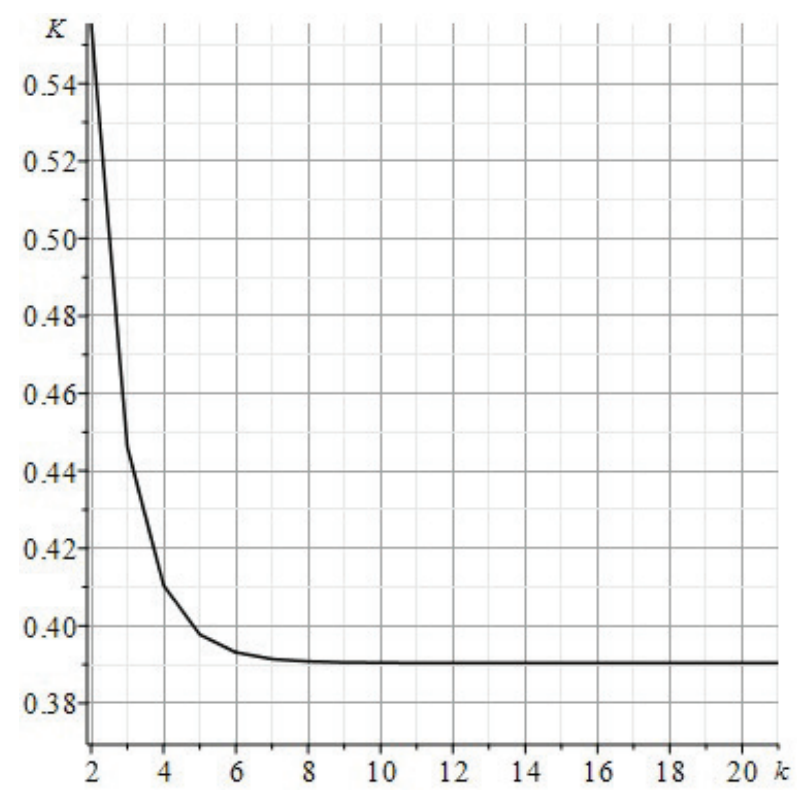

Fig. 2. Change in coefficient $K_{k}$ depending on the number of cycle $k$

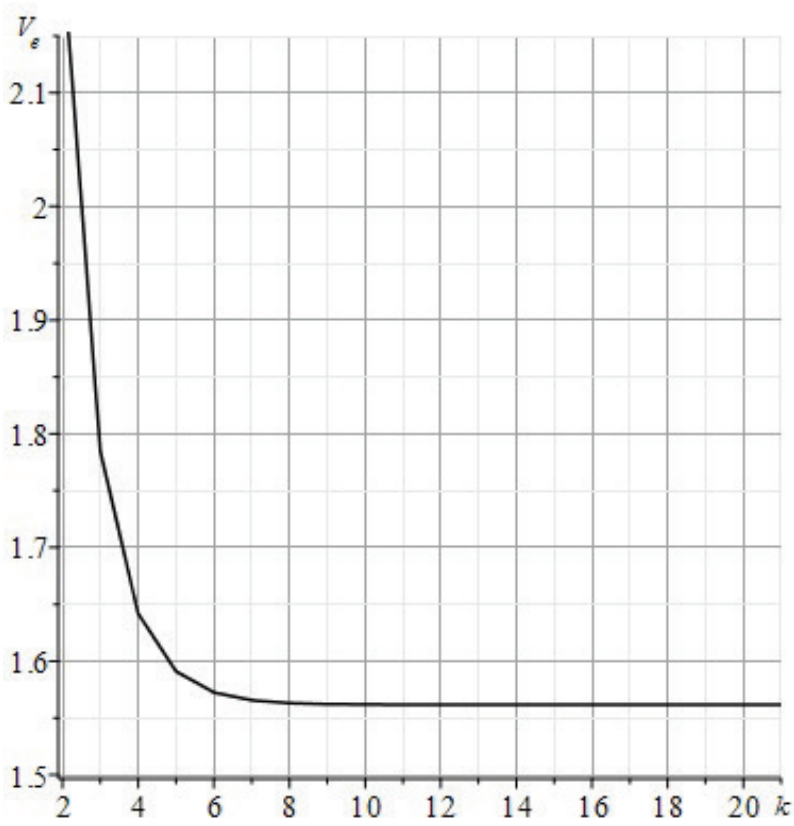

Fig. 3. $V_{\varepsilon}(k)$ values

Prospects of further studies lie in development of estimation algorithm with due account for available control actions and more complicated relationships in the wear of cutting tools used on various machine tools.

\section{References}

1. Z.Sh. Geyler, Self-tuning active control systems (Machine Building, Moscow, 1978)

2. A.N. Gavrilov (red.), Precision of production in mechanical engineering and instrument making (Machine Building, Moscow, 1973)
3. S.S. Volosov, Fundamentals of precision dimensional active control (Machine Building, Moscow, 1969)

4. M.S. Nevelson, Automatic control of metalworking accuracy (Machine Building, Leningrad, 1973)

5. V.Ya. Kopp, A.A. Skidan, A.I. Balakin, O.V. Filipovich, Analysis of differential entropy in engineering measurements in mechanical engineering and instrument making, Proc. of the Odessa Polytechnic University, 1(27), PP. 214 - 219 (2007)

6. R.E. Kalman, A New Approach to Linear Filtering and Prediction, Transaction ASME Journal of Basic Engineering, 86, PP. 35-45 (1960).

7. A.V. Balakrishnan, Kalman Filtering Theory. (Optimization Software, Inc., 1984)

8. J.C. Spall, Introduction to Stochastic Search and Optimization: Estimation, Simulation, and Control (Hoboken, New Jersey: Wiley, 2003).

9. G. Welch, G. Bishop, An Introduction to the Kalman Filter (Los Angeles, 2001).

10. B.D.O. Anderson, J.B. Moore, Optimal Filtering (New York: Dover, 2005).

11. Yu.E. Voskoboinikov, Recursive estimation of the state vector of the dynamic system (Novosibirsk: NSTU, 2014)

12. Yu.E. Voskoboinikov, The divergence criterion and the algorithm for adapting the recurrent algorithm for estimating the state vector, Scientific bulletin of the NSTU, 3(60), PP. 7-22 (2015)

13. R.G. Brown, P.Y.C. Hwang, Introduction to Random Signals and Applied Kalman Filtering, Second Edition (John Wiley \& Sons, Inc., 1992)

14. A. Gelb, Applied Optimal Estimation (MIT Press, Cambridge, MA., 1974)

15. Mohinder S. Grewal, P. Andrews Angus, Kalman Filtering Theory and Practice (Upper Saddle River, NJ USA, Prentice Hall, 1993)

16. O.L.R. Jacobs, Introduction to Control Theory, 2nd Edition (Oxford University Press, 1993)

17. Richard Lewis, Optimal Estimation with an Introduction to Stochastic Control Theory (John Wiley \& Sons, Inc., 1986).

18. Peter S. Maybeck, Stochastic Models, Estimation, and Control (Academic Press, Inc., 1979)

19. J.C Bertein, R. Ceschi, Processus stochastiques et filtrage de Kalman (Hermes, 1998)

20. C.K. Chui, G. Chen, Kalman Filtering (SpringerVerlag, 1987)

21. Mourad Barkat, Signal Detection and Estimation (Artech House Inc, 2005)

22. I.N. Sinitsyn, Kalman and Pugachev Filters (Moscow, 2006) 